\title{
Barriers of Asthma Care among Asthmatic Children in Saudi Arabia: Maternal Perspectives
}

\author{
Abeer Alatawi ${ }^{*}$, Meshaal Alanazi ${ }^{2}$ \\ ${ }^{1}$ Department of Nursing, Faculty of Applied Medical Sciences, Tabuk University, Tabuk, Saudi Arabia \\ ${ }^{2}$ Department of Emergency Medical Services, Saudi Red Crescent Authority, Tabuk, Saudi Arabia \\ Email: *asalatawi@ut.edu.sa
}

How to cite this paper: Alatawi, A. and Alanazi, M. (2020) Barriers of Asthma Care among Asthmatic Children in Saudi Arabia: Maternal Perspectives. Open Journal of Pediatrics, 10, 302-313.

https://doi.org/10.4236/ojped.2020.102031

Received: April 20, 2020

Accepted: May 29, 2020

Published: June 1, 2020

Copyright $\odot 2020$ by author(s) and Scientific Research Publishing Inc. This work is licensed under the Creative Commons Attribution International License (CC BY 4.0).

http://creativecommons.org/licenses/by/4.0/

\section{(c) (i) Open Access}

\begin{abstract}
Background Bronchial asthma is one of the most common chronic conditions among children. Despite the improvement in asthma treatment regimens, its prevalence and related morbidity are increasing, especially among underserved, minority children. There are barriers in the management of asthma, which may impact the quality of outcomes. The goal of this study is to explore these barriers. Methods A cross-sectional study was conducted on interview data collected through 2019 from mothers of children (aged 6 - 12 years) with asthma visiting, for convenience, a public shopping mall. The interviewees were randomly selected, because they met the inclusion criteria. Participants were considered if the mother answered "yes" to the following questions: Has your child had physician-diagnosed asthma? Is your child currently taking asthma medications of asthma? Two community nurses conducted the interviews. Data was obtained using administrative questionnaires. Data were analyzed using SPSS version 21.0 (IBM Inc., Chicago, IL, USA). Descriptive statistics of percentage, mean, frequency and standard deviation were applied for categorical and continuous variables. Results Three hundred mothers participated in this study. Their average age was 36.8 years, $55 \%$ were housekeepers by profession, and $34 \%$ had obtained less than high school education. The majority of children (61\%) were males, $45 \%$ had moderate asthma, and $42 \%$ had mild asthma. The most frequent types of barriers identified by parents were environmental factors $(67.7 \%)$, followed by health care providers (63\%), the health care system (48\%), and patient or family characteristics (43\%). Mothers were specifically concerned about the use, safety, and long-term complications of medications, the impact of exercise limitation on their child's quality of life, and their own quality of life. Conclusion This study showed several barriers against asthma care in Saudi Arabia, which mainly related to environmental or personal characteristics.
\end{abstract}


This highlights the need to enhance current policies within the health care system in Saudi Arabia to overcome these barriers.

\section{Keywords}

Barriers to Asthma Care, Mothers, Children, and Saudi Arabia

\section{Background}

Asthma is a chronic pulmonary disorder affecting millions of individuals around the world [1]. The disorder is characterized by wheezing, shortness of breath, and coughing. Previous research has remarked that asthma is the most common pediatric disease. Yet, the burden of asthma is often disregarded, because the mortality rate is lower than that of other respiratory disorders, such as pneumonia [2]. The Global Initiative for Asthma Report estimates asthma currently affects 300 million individuals. When the disease is uncontrolled, it reduces the quality of life, restricts participation in certain activities, and may cause death. Hence, it is considered a severe health problem worldwide. Internationally, the estimated prevalence of asthma in different countries is between $1 \%-18 \%$ [1] [2].

In Saudi Arabia, it is estimated that more than 2 million Saudis have asthma, and the prevalence in children is $8 \%-25 \%$ [3], the highest prevalence (25\%) reported by physicians in 2004 [4] [5]. Morbidity and mortality due to asthma have increased significantly and intensified public health concerns [6]. Bronchial asthma does not only affect the physiological function of the respiratory system, but it may also affect the individual's quality of life, leading to missing days from school or work, emergency hospital visits, hospitalization, and caregivers and parents' time and effort [6] [7] [8].

Multiple influences might contribute to the prevalence of advanced asthma and the severity of symptoms among children [9]. Health care system features are those related to whether parents have access to suppliers or health care, founded on insurance appearance and category. Although a number of these issues have been proposed to serve as barriers to optimal asthma management and good health outcomes, there are inadequate data concerning parental perceptions of barriers to ideal asthma care for children and families [10] [11].

While barriers to asthma management amongst adults are well documented, the barriers for children with asthma are not well understood. The purpose of this study was to identify and discuss the challenges of maternal care of asthmatic children that affect their ability to control or prevent their children's asthma symptoms.

\section{Method}

\subsection{Study Design and Setting}

A cross-sectional study was conducted between August and October 2019 in 
Tabuk, Saudi Arabia. The participants were mothers of children with asthma aged 6 - 12 years visiting a public area (shopping mall) who were willing to participate in the study after the purpose of the research was explained, and they were assured their responses would be anonymous and confidential. Written informed consent was obtained from the participants.

\subsection{Recruitment of Study Participants}

The sample included 300 mothers of children, 6 - 12 years old, who had physician-diagnosed asthma. Purposive sampling in consecutive periods was utilized. Purposive sampling involves the selection of readily available participants [12]. This sample was convenient, though random, because there were inclusion criteria. Interviewees were considered if the mother answered "yes" to the following questions about asthma: Has your child had physician-diagnosed asthma? Is your child currently taking asthma medication? Two community nurses conducted the interviews. A postcard was provided that allowed mothers to decline participation. To maximize participation, mothers who did not decline participation were contacted by telephone to determine interest and eligibility. No incentives were provided for participating.

\subsection{Inclusion/Exclusion Criteria}

Inclusion criteria for mothers included the following: having a child between 6 and 12 years old who had physician-diagnosed asthma and experienced asthma symptoms in the preceding 24 months. Mothers were excluded if their child had any chronic medical disease in addition to asthma. An enrollment questionnaire was used to determine demographics and asthma severity.

\subsection{Study Questionnaire}

Questions on the severity of disease were based on the criteria established by the Nationwide Heart, Lung, and Blood Institute's Guidelines for the Diagnosis and Management of Asthma [13]. A self-administered questionnaire was used previously to assess the parental barriers concerning asthma and its management in their children [14]. Subsequently, the questionnaire was pilot-tested among 40 mothers. Slight alterations were completed after the pilot inspection to assurance its appropriateness and consistency. This internal consistency reliability of the questionnaire was computed statistically by Cronbach's alpha test, the result of which was over 0.7. Then, the questionnaire was translated into Arabic to enable the participants to answer the questions. After translation and back translation, the content validity of the questionnaire was supported by five experts from multiple fields related to nursing and public health.

The researchers consistently employed close-ended questions. Two community nurses assisted to conduct the interviews with the mothers. The questionnaire was composed of two sections, the first of which was designed to accumulate socio-demographic information of the participant mothers and their child- 
ren. The second part was allocated to collect information about group and subgroup barriers with incidence data to include patient/family factors, health care system factors, health care provider factors, and environmental factors.

\subsection{Statistical Analysis}

SPSS version 21.0 (IBM Inc., Chicago, IL, USA) was used for data analysis. Descriptive statistics of percentage, mean, frequency and standard deviation were applied to categorical and continuous variables. Pearson's chi-squared test was used to compare the distribution of categorical variables. The t-test was applied to investigate the differences between variables.

\section{Results}

\subsection{Maternal Socio-Demographic Characteristics}

Three hundred mothers participated in this study. The largest mean age group was 31 - 40 years $(58 \%, 174 / 300)$, followed by 20 - 30 years $(40.3 \%, 121 / 300)$, and $<20$ years $(1.7 \%, 5 / 300)$. In addition, 55\% (165/300) of the mothers were housekeepers (Table 1). Regarding formal education, 34\% (102/300) of the mothers had less than high school, and 19.3\% (58/300) had more than high school. Also, $54 \%(164 / 300)$ have less than three from family members.

Table 1. Socio-demographic characteristics of participant mothers.

\begin{tabular}{|c|c|}
\hline Characteristics & $\mathrm{N}(\%)$ \\
\hline \multicolumn{2}{|l|}{ Age (Years) } \\
\hline $20-30$ & $121(40.3)$ \\
\hline $31-40$ & $174(58.0)$ \\
\hline$<20$ & $5(1.7)$ \\
\hline \multicolumn{2}{|l|}{ Mother occupation } \\
\hline Employee & $135(45)$ \\
\hline Housekeeper & $165(55)$ \\
\hline \multicolumn{2}{|l|}{ Educational level } \\
\hline High school & $80(26.7)$ \\
\hline Less than high school & $102(34.0)$ \\
\hline More than high school & $58(19.3)$ \\
\hline University & $60(20.0)$ \\
\hline \multicolumn{2}{|l|}{ Number of family members } \\
\hline $4-7$ & $136(45.3)$ \\
\hline$<3$ & $164(54.7)$ \\
\hline
\end{tabular}




\subsection{Clinical Characteristics of Children}

More than half of the children were male $(61.3 \%, 184 / 300)$, and $48.3 \%(145 / 300)$ received asthma care at the hospital/community. In addition, $45 \%(135 / 300)$ of patients had moderate asthma, and 13\% (39/300) had severe asthma. Approximately half of them had suffered from asthma for less than two years, and $47 \%$ $(141 / 300)$ of children visited the hospital less than two times per month. Most of the patients practice medication compliance (Table 2).

\subsection{Categories and Subcategories of Barriers with Frequency/Percent}

The greatest number of mothers (67.7\%) considered environmental factors to be barriers to asthma care. The greatest barrier reported among environmental subcategories (96.7\%) was "the mother has knowledge regarding environmental triggers for asthma (includes aeroallergens, dust, cockroaches, exercise, weather)/perceptions regarding the role of environmental triggers for asthma." "Housing constraints-unable to move/change allergen exposure" was the least reported environmental factor (10.3\%).

Table 2. Characteristics of participant children.

\begin{tabular}{|c|c|}
\hline Characteristics & $\mathrm{N}(\%)$ \\
\hline \multicolumn{2}{|l|}{ Gender } \\
\hline Female & $116(38.7)$ \\
\hline Male & $184(61.3)$ \\
\hline \multicolumn{2}{|l|}{ Usual care site for asthma } \\
\hline Clinic & $105(35)$ \\
\hline Hospital/community & $145(48.3)$ \\
\hline Private office & $50(16.7)$ \\
\hline \multicolumn{2}{|l|}{ Asthma severity } \\
\hline Mild & $126(42)$ \\
\hline Moderate & $135(45)$ \\
\hline Severe & $39(13)$ \\
\hline \multicolumn{2}{|c|}{ Duration of asthma (years) } \\
\hline $3-6$ & $138(46)$ \\
\hline$<2$ & $147(49)$ \\
\hline$>6$ & $15(5)$ \\
\hline \multicolumn{2}{|c|}{ Number of hospital visits per month } \\
\hline $3-5$ & $109(36.3)$ \\
\hline$<2$ & $141(47)$ \\
\hline$>5$ & $50(16.7)$ \\
\hline \multicolumn{2}{|c|}{ Compliance with medication } \\
\hline No & $2(0.7)$ \\
\hline Yes & $298(99.3)$ \\
\hline
\end{tabular}


The second most reported category (63\%) of maternal concern was related to the health care provider, with the subcategories "waiting time in the office at the time of appointment" reported by $98 \%$ of mothers and "availability of primary provider/representative after office hours" reported by only $16 \%$. The third most reported category (48\%) was the health care system, with most (91\%) mothers reporting the subcategory "the mother has good knowledge regarding insurance/Medicaid" and the least (4.3\%) reporting "changes in insurance policies regarding medication coverage/methods of obtaining medications". The least reported (43\%) category was patient or family factors, with the majority of mothers (98.7\%) reporting "knowledge about asthma, asthma medications, and their use was good" and a small proportion (6.3\%) reporting "its lack of conflict with children". The frequency of comments coded for each subcategory is shown in Table 3.

Statistics for categories ranged between 0.83 and 0.84 for each pair of independent raters, indicating excellent agreement for the classification by category. Because agreement by subcategory is conditional on agreement by category, percent agreement was used to assess agreement between raters for each subcategory (Table 3). Family attitudes and beliefs and their environment modify how mothers respond to the barriers in caring for their children with asthma. Concerning health care provider factors, mothers were more likely to report that too long of wait time in the office for an appointment. Within types of care, there were no differences in barriers by asthma severity.

Table 3. Categories and subcategories of barriers with frequency/percent.

\begin{tabular}{ll}
\hline Category 1 Patient/Family Factors & \\
\hline Number of caretakers/number of primary households & N (\%) \\
\hline 1.0 & $109(36.3)$ \\
2.0 & $120(40)$ \\
3.0 & $48(16)$ \\
4.0 & $23(7.7)$ \\
Identified primary caretaker & \\
Father & $12(4)$ \\
Mother & $282(94)$ \\
Other & $6(2)$ \\
3) Lack of conflict with children & $19(6.3)$ \\
4) Priority of caring for asthma relative to other concerns & $94(31.3)$ \\
5) Compliance of family with doctor prescribed plan was good & $293(97.7)$ \\
6) Compliance of child with mother's recommendations was good & $270(90)$ \\
7) Mothers have positive attitude toward disease & $284(94.7)$ \\
8) Child has positive attitude toward disease & $266(88.7)$ \\
9) Family support for care/management of disease & $275(91.7)$ \\
10) Knowledge about asthma, asthma medications, and their use was good & $296(98.7)$ \\
Total & $\mathbf{1 8 2 6}(43.5)$ \\
\hline
\end{tabular}


Continued

Category 2 Health Care System Factors

11) The primary location for asthma care

community clinic

$41(13.7)$

hospital clinic

$211(70.3)$

specialist

$48(16)$

12) Presence of insurance

13) Changes in insurance policies regarding medication coverage/methods of obtaining medications

14) The mother has good knowledge regarding insurance/Medicaid

Total

Category 3 Health Care Provider Factors

15) General satisfaction with health care provider was trust

16) Relationship between health care provider and mother/child is good

17) Education regarding asthma, asthma care, and medication use is given in an understandable manner

18) Type of health care provider seen to asthma care

Nurse practitioner

Others

Primary care doctor

19) Continuity of provider

20) Availability of primary provider during office hours

21) Availability of primary provider/representative after office hours

22) Ability to get an appointment for illness in a timely fashion

23) Waiting time in the office at the time of appointment

Category 4 Environmental Factors

24) Accessibility to provider/available and reliable transportation/geographic location of the provider

25) Financial constraints-money for transportation and medications

26) Housing constraints-unable to move/change allergen exposure

27) Accessibility to a pharmacy-open, safe location 24 hours/day

28) Available social support from friends and community?

29) Available support from school

30) The mother has knowledge regarding environmental triggers for asthma (includes aeroallergens, dust, cockroaches, exercise, weather)/perceptions 


\section{Discussion}

The current study aimed to explore the barriers of asthma care among mothers of asthmatic children. The mother's replies to the questionnaire were organized into four overarching categories: patient/family factors barriers, environmental barriers, health care provider barriers, and health care system barriers. Citing access to medical care, health insurance, and continuity of care as the significant barriers to quality asthma care for children, mothers most frequently reported extrinsic environmental factors (67.7\%) and health care providers (63\%). These results are consistent with those of another study [14]. This study reinforces the need to expand asthma management beyond a strictly biomedical approach to one that incorporates environmental control. Biomedical advancements expected to refine quality asthma care are deficient in environmental control, financial limitations such as money for transportation and medications, and accommodation restrictions. Other studies found that effective management of childhood asthma requires a comprehensive approach and involves a "therapeutic partnership" between the parents and the healthcare provider [15] [16].

Other studies have documented that traditional access to procedures such as the standard foundation of medical care does not, by itself, passably quantity a child's receipt of acceptable asthma management [17] [18]. Successful asthma care outcomes require collaborative work between physicians, health facilities, patients, and their families. Most parents described a standard source of care (97\%), and many parents described the particular characteristics of the site or provider as limiting the quality of asthma care. Access to care is a critical barrier for parents in our study, and those factors related to quality-of-life issues are critical to improving health consequences [19]. Similarly, studies reported that waiting too long for an appointment, unavailability of needed care, and inconvenient hours suggest that many barriers are related to the health care service infrastructure in the inner city, where evening appointment hours and telephone advice are typically lacking. One of the findings of this study was the strong correlation demonstrated between these barriers and asthma management [20].

In our study, patient and family features were perceived to provide the least asthma barriers; most mothers have strong opinions and arrogances about asthma care and management. Similarly, investigators in the National Cooperative Inner City Asthma Study observed the psychosocial variables that may distress asthma morbidity [10]. The conceptual framework was used to determine the association between psychosocial features and morbidity, integrates caretakers' arrogances and views as the main constituent of asthma management. These views were conceptualized within a biomedical context and directly related to caretakers' opportunities or self-efficacy concerning asthma management behaviors, such as observing medical arrangements and treatment processes. A study in Saudi Arabia argued that although it is necessary to address parents' health needs in asthma management and assess parents' perceptions of their asthma management assistance, that framework did not specifically address maternal 
health views, as recognized in our study [21].

What is particularly significant is that parents of younger children and teenagers permitted, unfortunately, entrustment of asthma self-management to children too young, too poorly qualified, or too unmotivated to manage their condition [22] [23] properly. Some mothers in our study permitted children to be accountable for regulating medicines at their leisure or not to take it at all. This is different from what has been found in other studies [24] [25]. Wade et al. [26] and McQuaid et al. [27] found that mothers stimulated responsible asthma self-management in children but continued to be aggressively involved in management as needed. It is critical for health care providers to be culturally sensitive to the family's needs to integrate alternative practices to aid more open physician-patient interactions and better management of pediatric asthma [28]. Since some caregivers felt stress and barriers affiliated with health care providers, improved healthcare provider/caregiver communication may help to mediate the barrier of provider stress in pediatric asthma care [29] [30].

The results of this study might be useful for healthcare professionals, healthcare administrators, and policy makers, in order to design educational programs for mothers of asthmatic children to improve their quality of life after determining areas of barriers for optimal asthma care. This is due to the fact that caregivers have major roles in managing asthma in children. This study also provided baseline data regarding Saudi mothers' whose the caregivers, their challenges about asthma care, which may encourage other researchers to conduct further research studies in this regard to confirm these findings, and find more conclusive findings by assessing knowledge about asthma among larger sample sizes and including more representative samples of the Saudi population.

\section{Limitations of the Study}

This study had several limitations. First, many independent factors that may affect the barriers of asthma care among mothers were not studied. Hereafter, further studies are recommended to exclude potential confounding factors. Second, the study did not include control groups of mothers in health care centers or hospitals to compare results. Participants recruited through a public area may not be generalizable to other populations. To better understand such effects, performing a case-control study that includes a control group from health care centers is recommended. Third, the nature of the cross-sectional design limits the cause-and-effect relationship. More studies are needed in larger, more diverse populations to determine if the identified barriers apply to other families of children with asthma.

\section{Conclusion}

This study offers vital information about the perspectives of barriers to asthma care among mothers of asthmatic children. It also establishes that mothers' explanations of barriers to asthma care differ dramatically from those customarily 
proposed by health care providers and academic researchers. This inequality in fictional barriers may help to describe why numerous children from disadvantaged backgrounds have less than ideal asthma health consequences. Health care providers, child health researchers, and advocates need to assess environmental factors and identify the barriers mothers identify as essential to progress health consequences for asthmatic children.

\section{Declarations}

Researchers certify that this research submitted for the publication in the result of own research and that this research (or any of its parts) has not been submitted from any other previous works to any other university or institution.

\section{Ethics Approval and Consent to Participate}

The ethics committee within the Health Affairs in Tabuk approved the study. All participants provided signed consent for their participation and publication. The manuscript does not report on or involve the use of any animal or human data or tissue.

\section{Consent for Publication}

The consent for publication is signed.

\section{Availability of Data and Materials}

The data that support the findings of this study are available, but restrictions apply to the availability of these data. They were used under privacy for the current study, and so are not publicly available. Data are, however, available from the authors upon reasonable request.

\section{Funding}

There was no funding for this study.

\section{Acknowledgements}

The ethics committee is appreciated for their cooperation and providing the opportunity to conduct this study. Also, we are grateful to all the mothers who participated in this study.

\section{Conflicts of Interest}

The authors declare no conflicts of interest regarding the publication of this paper.

\section{References}

[1] Vincent, S.D., Toelle, B.G., Aroni, R.A., Jenkins, C.R. and Reddel, H.K. (2006) "Exasperations" of Asthma: A Qualitative Study of Patient Language about Worsening Asthma. The Medical Journal of Australia, 184, 451-454. 
https://doi.org/10.5694/j.1326-5377.2006.tb00317.x

[2] Masoli, M., Fabian, D., Holt, S. and Beasley, R. (2004) Global Initiative for Asthma (GINA) Program. The Global Burden of Asthma: Executive Summary of the GINA Dissemination Committee Report. Allergy, 59, 469-478.

https://doi.org/10.1111/j.1398-9995.2004.00526.x

[3] Nasser, A., Saad, A.S. and Abdullah, T. (1997) Impact of Asthma Educational Program on Asthma Knowledge of General Practitioners. Annals of Saudi Medicine, 17, 550-552. https://doi.org/10.5144/0256-4947.1997.550

[4] Sobki, S.H. and Zakzouk, S.M. (2004) Point Prevalence of Allergic Rhinitis among Saudi Children. Rhinology, 42, 137-140.

[5] Al Frayh, A.R., Shakoor, Z., Gad El Rab, M.O. and Hasnain, S.M. (2001) Increased Prevalence of Asthma in Saudi Arabia. Annals of Allergy, Asthma \& Immunology, 86, 292-296. https://doi.org/10.1016/S1081-1206(10)63301-7

[6] Wa Somwe, S., Jumbe-Marsden, E., Mateyo, K., et al. (2015) Improving Paediatric Asthma Care in Zambia. Bulletin of the World Health Organization, 93, 732-736. https://doi.org/10.2471/BLT.14.144071

[7] Stewart, W.F., Ricci, J.A., Chee, E. and Morganstein, D. (2003) Lost Productive Work Time Costs from Health Conditions in the United States: Results from the American Productivity Audit. Journal of Occupational and Environmental Medicine, 45, 1234-1246. https://doi.org/10.1097/01.jom.0000099999.27348.78

[8] Al-Mobeireek, A. (2003) Prescribing for Asthmatic Children in Primary Care. Are We Following the Guidelines? Saudi Medical Journal, 24, 1274.

[9] Dashash, N.A. and Mukhtar, S.H. (2003) Prescribing for Asthmatic Children in Primary Care. Are We Following Guidelines? Saudi Medical Journal, 24, 507-511.

[10] Leiria Pinto, P., Cordeiro, M. and Pinto, R. (1999) Adolescents and School Asthma Knowledge and Attitudes. Allergologia et Immunopathologia, 27, 245-253.

[11] Wade, S., Weil, C. and Holden, G. (1997) Psychosocial Characteristics of Innercity Children with Asthma: A Description of the NCICAS Psychosocial Protocol. Pediatric Pulmonology, 24, 263-276. https://doi.org/10.1002/(SICI)1099-0496(199710)24:4<263::AID-PPUL5>3.0.CO;2$\underline{\mathrm{L}}$

[12] Cooper, D. and Schindler, P. (2011) Business Research Methods. 11th Edition, McGraw Hill, Boston.

[13] (1997) Expert Panel Report II. Guidelines for the Diagnosis and Management of Asthma/National Asthma Education and Prevention Program. National Asthma Education and Prevention Program, National Heart, Lung, and Blood Institute, National Institutes of Health, US Department of Health and Human Services, Bethesda.

[14] Mansour, M.E., Lanphear, B.P. and DeWitt, T.G. (2000) Barriers to Asthma Care in Urban Children: Parent Perspectives. Pediatrics, 106, 512-519.

https://doi.org/10.1542/peds.106.3.512

[15] Abu-Shaheen, A.K., Nofal, A. and Heena, H. (2016) Parental Perceptions and Practices toward Childhood Asthma. BioMed Research International, 2016, Article ID: 6364194. https://doi.org/10.1155/2016/6364194

[16] Zaraket, R., Al-Tannir, M.A., Bin Abdulhak, A.A., Shatila, A. and Lababidi, H. (2011) Parental Perceptions and Beliefs about Childhood Asthma: A Cross-Sectional Study. Croatian Medical Journal, 52, 637-643. https://doi.org/10.3325/cmj.2011.52.637

[17] Abu-Shaheen, A., Alfayyad, I., Nofal, A., et al. (2018) Perceptions and Practices in 
Parents of Saudi Children with Asthma: A Cross-Sectional Survey. Cureus, 10, e2213. https://doi.org/10.7759/cureus.2213

[18] Hazir, T., Das, C., Piracha, F., Waheed, B. and Azam, M. (2002) Carers' Perception of Childhood Asthma and Its Management in a Selected Pakistani Community. Archives of Disease in Childhood, 87, 287-290. https://doi.org/10.1136/adc.87.4.287

[19] Andrews, L., Lokuge, S., Sawyer, M., Lillywhite, L., Kennedy, D. and Martin, J. (1998) The Use of Alternative Therapies by Children with Asthma: A Brief Report. Journal of Paediatrics and Child Health, 34, 131-134. https://doi.org/10.1046/j.1440-1754.1998.00179.x

[20] Crain, E.F., Kercsmar, C., Weiss, K.B., Mitchell, H. and Lynn, H. (1998) Reported Difficulties in Access to Quality Care for Children with Asthma in the Inner City. Archives of Pediatrics and Adolescent Medicine, 152, 333-339. https://doi.org/10.1001/archpedi.152.4.333

[21] Al-Binali, A.M., Mahfouz, A.A., Al-Fifi, S., Naser, S.M. and Al-Gelban, K.S. (2010) Asthma Knowledge and Behaviours among Mothers of Asthmatic Children in Aseer, South-West Saudi Arabia. Eastern Mediterranean Health Journal, 16, 1153-1158. https://doi.org/10.26719/2010.16.11.1153

[22] BinSaeed, A.A. (2014) Caregiver Knowledge and Its Relationship to Asthma Control among Children in Saudi Arabia. Journal of Asthma, 51, 870-875. https://doi.org/10.3109/02770903.2014.906608

[23] Trent, C.A., Zimbro, K.S. and Rutledge, C.M. (2015) Barriers in Asthma Care for Pediatric Patients in Primary Care. Journal of Pediatric Health Care, 29, 70-79. https://doi.org/10.1016/j.pedhc.2014.07.002

[24] Kornblit, A., Cain, A., Bauman, L.J., Brown, N.M. and Reznik, M. (2018) Parental Perspectives of Barriers to Physical Activity in Urban Schoolchildren with Asthma. Academic Pediatrics, 18, 310-316. https://doi.org/10.1016/j.acap.2017.12.011

[25] Sadreameli, S.C., Riekert, K.A., Matsui, E.C., Rand, C.S. and Eakin, M.N. (2018) Family Caregiver Marginalization Is Associated with Decreased Primary and Subspecialty Asthma Care in Head Start Children. Academic Pediatrics, 18, 905-911. https://doi.org/10.1016/j.acap.2018.04.135

[26] Wade, S.L., Islam, S., Holden, G., Kruszon-Moran, D. and Mitchell, H. (1999) Division of Responsibility for Asthma Management Tasks between Caregivers and Children in the Inner City. Journal of Developmental \& Behavioral Pediatrics, 20, 93-98. https://doi.org/10.1097/00004703-199904000-00004

[27] McQuaid, E.L., Penza-Clyve, S.M. and Nassau, J.H. (2001) The Asthma Responsibility Questionnaire: Patterns of Family Responsibility for Asthma Management. Children's Health Care, 30, 183. https://doi.org/10.1207/S15326888CHC3003_2

[28] Laster, N., Holsey, C.N., Shendell, D.G., McCarty, F.A. and Celano, M. (2009) Barriers to Asthma Management among Urban Families: Caregiver and Child Perspectives. Journal of Asthma, 46, 731-739. https://doi.org/10.1080/02770900903082571

[29] AlOtaibi, E. and AlAteeq, M. (2018) Knowledge and Practice of Parents and Guardians about Childhood Asthma at King Abdulaziz Medical City for National Guard, Riyadh, Saudi Arabia. Risk Management and Healthcare Policy, 11, 67-75. https://doi.org/10.2147/RMHP.S143829

[30] Al-Anazi, A., Al Moamary, M., Ismaeli, T., et al. (2015) Asthma in the Pediatric Population: Level of Perception among the Parents and Guardians. International Journal of Medicine and Public Health, 5, 14-18. https://doi.org/10.4103/2230-8598.151240 